\title{
Harvesting Waste Thermal Energy Using a Surface-Modified Carbon Fiber-Based Thermo-Electrochemical Cell
}

\author{
Denis Artyukhov ${ }^{1}\left(\mathbb{D}\right.$, Nikolay Kiselev ${ }^{1,2,3}{ }^{\mathbb{D}}$, Nikolay Gorshkov ${ }^{1} \mathbb{D}$, Natalya Kovyneva ${ }^{1}$, Olga Ganzha $^{1}$, \\ Maria Vikulova ${ }^{1}\left(\mathbb{D}\right.$, Alexander Gorokhovsky ${ }^{1}\left(\mathbb{D}\right.$, Peter Offor ${ }^{4,5}$, Elena Boychenko ${ }^{2,3} \mathbb{D}^{1}$ and Igor Burmistrov ${ }^{1,2,3, *}$
}

check for updates

Citation: Artyukhov, D.; Kiselev, N.; Gorshkov, N.; Kovyneva, N.;

Ganzha, O.; Vikulova, M.;

Gorokhovsky, A.; Offor, P.;

Boychenko, E.; Burmistrov, I

Harvesting Waste Thermal Energy Using a Surface-Modified Carbon Fiber-Based Thermo-Electrochemical Cell. Sustainability 2021, 13, 1377. https://doi.org/10.3390/su13031377

Academic Editor: Jose

Navarro Pedreño

Received: 8 December 2020

Accepted: 25 January 2021

Published: 28 January 2021

Publisher's Note: MDPI stays neutral with regard to jurisdictional claims in published maps and institutional affiliations.

Copyright: (c) 2021 by the authors. Licensee MDPI, Basel, Switzerland. This article is an open access article distributed under the terms and conditions of the Creative Commons Attribution (CC BY) license (https:// creativecommons.org/licenses/by/ $4.0 /)$.
1 Department of Chemistry and Chemical Technology of Materials, Yuri Gagarin State Technical University of Saratov, 410054 Saratov, Russia; mr.tokve@gmail.com (D.A.); nikokisely12345@gmail.com (N.K.); gorshkov.sstu@gmail.com (N.G.); k.natasha_86@bk.ru (N.K.); gangaolya@gmail.com (O.G.); vikulovama@yandex.ru (M.V.); algo54@mail.ru (A.G.)

2 Department of Functional Nanosystems and High-Temperature Materials, National University of Science and Technology «MISiS», 119049 Moscow, Russia; elena.boychenko.sar@gmail.com

3 Engineering Center, Plekhanov Russian University of Economics, 117997 Moscow, Russia

4 Metallurgical and Materials Engineering Department, University of Nigeria, Nsukka 410001, Nigeria; ykonukhov@misis.ru

5 Africa Centre of Excellence for Sustainable Power and Energy Development (ACE-SPED), University of Nigeria, Nsukka 410001, Nigeria

* Correspondence: burmistrov.in@misis.ru

\begin{abstract}
An important direction in the development of energy saving policy is harvesting and conversion into electricity of low-grade waste heat. The present paper is devoted to the improvement of the efficiency of thermo-electrochemical cells based on carbon fiber electrodes and potassium ferri-/ferrocyanide redox electrolyte. The influence of the carbon fiber electrode surface modification (magnetron deposition of silver and titanium or infiltration implantation of nanoscale titanium oxide) on the output power and parameters of the impedance equivalent scheme of a thermoelectrochemical cell has been studied. Two kinds of cell designs (a conventional electrochemical cell with a salt bridge and a coin cell-type body) were investigated. It was found that the nature of the surface modification of electrodes can change the internal resistance of the cell by three orders of magnitude. The dependence of the equivalent scheme parameters and output power density of the thermoelectric cell on the type of electrode materials was presented. It was observed that the maximum power for carbon fiber modified with titanium metal and titanium oxide was $25.2 \mathrm{~mW} / \mathrm{m}^{2}$ and the efficiency was $1.37 \%$.
\end{abstract}

Keywords: thermo-electrochemical cell; waste heat harvesting; carbon fiber; surface modification; efficiency

\section{Introduction}

The attention of the scientific community has recently been focused on generating the cheapest and cleanest energy. There are many areas where the amount of energy dissipated in the atmosphere is enormously high. This is especially significant in places where heat radiators and pipes with hot liquid are used. Moreover, a lot of potential heat is generated by mechanical and electrical equipment.

The harvest of low-temperature waste heat is a widely studied topic nowadays. Thermo-electrochemical cells (thermocells or thermo-galvanic cells, (TECs)) are one of the cheapest means to convert low-grade waste heat into electricity [1-4]. Typically, a TEC is based on a redox electrolyte and two electrodes placed at different temperatures [5-12]. The emergence of the temperature difference between the electrodes, in other words, the entropy difference between the two sides of the redox process, generates a potential difference and maintains a continuous flow of electric current in the TEC. 
The conversion process is based on the temperature coefficient of the electrode potential or hypothetical Seebeck coefficient of the cells. The output voltage is proportional to the temperature difference between hot and cold electrodes because of the difference in entropy of the redox process for hot and cold electrodes [2,3]. The hypothetical Seebeck coefficient of thermo-electrochemical cells can be calculated by the following formula [4]:

$$
S e=\frac{\delta V}{\delta T}=-\frac{\Delta S_{r x}^{0}}{n F}
$$

where $\delta V$ is the full-cell voltage, $\delta T$ is the interelectrode temperature difference, $\Delta S_{r x}^{0}$ is the entropy change for the cell reaction, $n$ is the number of electrons transferred in the reaction, and $F$ is Faraday's constant.

Currently, researchers are focused on the $\left[\mathrm{Fe}(\mathrm{CN})_{6}\right]^{3-} /\left[\mathrm{Fe}(\mathrm{CN})_{6}\right]^{4-}$ redox couple and carbon nanomaterials with high specific surface areas (carbon nanotubes and reduced graphene oxide) as they exhibit fast electron transfer kinetics and a hypothetical Seebeck coefficient of about $1.4 \mathrm{mV} / \mathrm{K}$. The highest reported Seebeck coefficient of $9.9 \mathrm{mV} / \mathrm{K}$ has been demonstrated for a thermocell containing acetone and iso-propanol as the redox couple, when the vaporization entropy of acetone increases the total entropy change in the conversion of iso-propanol to acetone [13]. The highest hypothetical Seebeck coefficient for aqueous electrolyte-based thermocells $(4.5 \mathrm{mV} / \mathrm{K})$ and, accordingly, high open-circuit voltage values of up to $0.2 \mathrm{~V}$ have been presented in studies [14,15].

The accumulation and storage of charge in electrochemical systems (capacitors, thermoelectrochemical cells) occur in the electric double-layer at the electrode/electrolyte junction. Therefore, electrode materials must meet the following requirements: high electrical conductivity, high specific surface area, availability of the porous structure to ions and electrolyte molecules, and low density. Carbon materials satisfy these requirements [16-20]. The relevance of carbon materials for electrochemical applications is due to the unique combination of chemical and physical properties: carbon electrodes are well polarized, stable over a wide range of temperatures, and chemically inert. In addition, they are characterized by high conductivity, high specific surface area, and relatively low cost and have a porous structure which can be controlled.

The best world records of TEC efficiency and power output were achieved with the catalytic modification of carbon nanotube aerogel by Pt nanoparticles [1]. In this work, a maximum power output of $6.6 \mathrm{~W} \mathrm{~m}^{2}$ and a Carnot relative efficiency of $3.95 \%$ described in the literature have been shown. Several studies have described that the addition of metal nanoparticles provides a significant catalytic effect and increases the current density in a continuous redox reaction in a system of $\left[\mathrm{Fe}(\mathrm{CN})_{6}\right]^{3-} /\left[\mathrm{Fe}(\mathrm{CN})_{6}\right]^{4-}[4,21,22]$.

Nevertheless, carbon nanotubes are still limited in industrial production, are extremely expensive, and require the use of polymer binders for the creation of electrode materials. Simultaneously, carbon fiber (CF) materials have a significant advantage because they do not require the addition of binding components (in comparison with polymer additives for CNTs and carbon black-based electrodes $[23,24])$ in the manufacture of electrodes. CF materials are obtained mainly on the basis of polyacrylonitrile (PAN) pitch and rayonbased precursor. Isotropic pitch and rayon-based carbon fibers are excellent materials for the production of activated carbon fibers with very high specific areas $\left(>1500 \mathrm{~m}^{2} / \mathrm{g}\right)$ and are likely to dominate as materials for liquid as well as gaseous adsorption and environmental protection.

Therefore, in the present work, materials based on commercially available carbon fiber modified by easily reproducible magnetron sputtering (with $\mathrm{Ti}$ and Ag coating) and infiltration methods (with $\mathrm{TiO}_{2}$ nanopowder) were investigated as cheap electrode materials for thermo-electrochemical cells based on the $\left[\mathrm{Fe}(\mathrm{CN})_{6}\right]^{3-} /\left[\mathrm{Fe}(\mathrm{CN})_{6}\right]^{4-}$ redox system, which can be easily scaled. 


\section{Materials and Methods}

\subsection{Materials}

For electrolyte preparation, $\mathrm{K}_{3}\left[\mathrm{Fe}(\mathrm{CN})_{6}\right](99.8 \%$ purity, CAS $13746-66-2)$ and $\mathrm{K}_{4}\left[\mathrm{Fe}(\mathrm{CN})_{6}\right]$ (99.8\% purity, CAS 14459-95-1) were purchased from Sigma-Aldrich GmbH (Schnelldorf, Germany) and used without further purification. Ferri-/ferrocyanide redox electrolyte in the ratio of 1:1 with a total concentration of $0.3 \mathrm{~mol} / \mathrm{L}$ in distilled water was used as the base.

Carbon fiber Busofit TM-04 (Busofit) commercial products were purchased from Svetlogorsk Khimvolokno OJSC (Svetlogorsk, Republic of Belarus), with a diameter of the initial fiber of about 6-8 $\mu \mathrm{m}$ and an electrical resistivity of approximately $20-200 \Omega \mathrm{m}$.

Four modifications of Busofit carbon fabric were used as electrodes. Modification was carried out by vacuum sputtering of titanium using magnetron sources and condensing its vapors onto a carbon cloth tape:

1. Basic Busofit;

2. Busofit with Ag magnetron sputtering;

3. Busofit with Ti magnetron sputtering;

4. Busofit with infiltrated dispersion of $\mathrm{TiO}_{2}$ nanoparticles.

The dispersal of titanium oxide (from a $5 \%$ dispersion of $\mathrm{TiO}_{2}$ ) into Busofit fabric was carried out in vacuum, in a Buchner filter on a Bunsen funnel. Then, the coating was dried for $1 \mathrm{~h}$ at temperatures of 80 and $150{ }^{\circ} \mathrm{C}$.

For cells in the coin cell CR2025, an industrial separator for Li-ion current sources was used.

\subsection{Measurement and Characterization Technique}

The potentiodynamic method was used to obtain the maximum power values. To determine the voltage of the open circuit of the thermal cell on the cold and hot sides, the following temperature differences were consistently created: 10, 20, 30, 40, and $50 \mathrm{~K}$. After some time, a stable potential was established in the system, and the value of this potential was taken as the open circuit voltage $\left(\mathrm{U}_{\mathrm{OC}}\right)$, which is unique to each material and each temperature. After that, the cell was discharged by creating a reverse potential, increasing from $0 \mathrm{mV}$ to the open circuit voltage ( $\mathrm{U}_{\mathrm{OC}}$, which was established in the previous step). $\mathrm{U}_{\mathrm{OC}}$ was increased at the rate of $1 \mathrm{mV} / \mathrm{s}$. During all experiments, the current flowing through the cell was recorded.

One of the stages of processing the measurement results was the construction and analysis of an equivalent scheme (ES), i.e., electrical circuits having the same frequency dependence on impedance as the studying cell. It can be used to determine the structural features of the investigated systems (electrode-electrolyte heterojunctions, particle coupling in clusters, surface and bulk conductivities), microscopic parameters (cluster sizes and their local resistance), and other characteristics of the objects under study.

A typical ES consists of several different elements: resistors, capacitors, inductors, Warburg impedance, and constant phase shift element [8]. Analysis of the ES and calculation of the total resistance are associated with the addition of harmonic oscillations of currents and voltages. In case of studying the dependence of the output parameters on the presence and properties of separators, the most interesting is Warburg's impedance (W), which characterizes diffuse processes inside the cell.

The main method of evaluating the data obtained by the impedance spectroscopy technique is the analysis of impedance hodographs, i.e., by studying the dependence of the imaginary parts of permittivity on the real parts of one set of Nyquist coordinates. Based on the data obtained from the hodograph, a cell's equivalent scheme can be constructed [25].

\section{Results and Discussion}

In the present work, two kinds of TEC architecture were investigated: thermocells with a salt bridge and thermocells with the coin cell CR2025 case. Both types have advantages and disadvantages, so the comparison was very informative. 
Systems with a salt bridge are easy to maintain and can be precisely controlled under persistent large temperature differences. This provides an accurate measurement of the Seebeck coefficient. Systems in the coin cell CR2025 have two important application benefits: the assembly speed and the reproducibility of the results for cells in this type of case are much better than in other types of electrochemical systems.

Modification of Busofit-based electrode materials for both types of cells was carried out for two reasons. The first was for the formation of electrodes with a minimum contact resistance of the metal layer-Busofit. The second is a decrease in contact resistance between the Busofit layers, which determines the capacity of the electrolytic cell. In this case, the metal layer, in addition to low electrical resistance, must have a high porosity, which ensures the penetration of the electrolyte between the layers of Busofit and increases the total porosity. SEM images of the Busofit materials are shown in Figure 1.

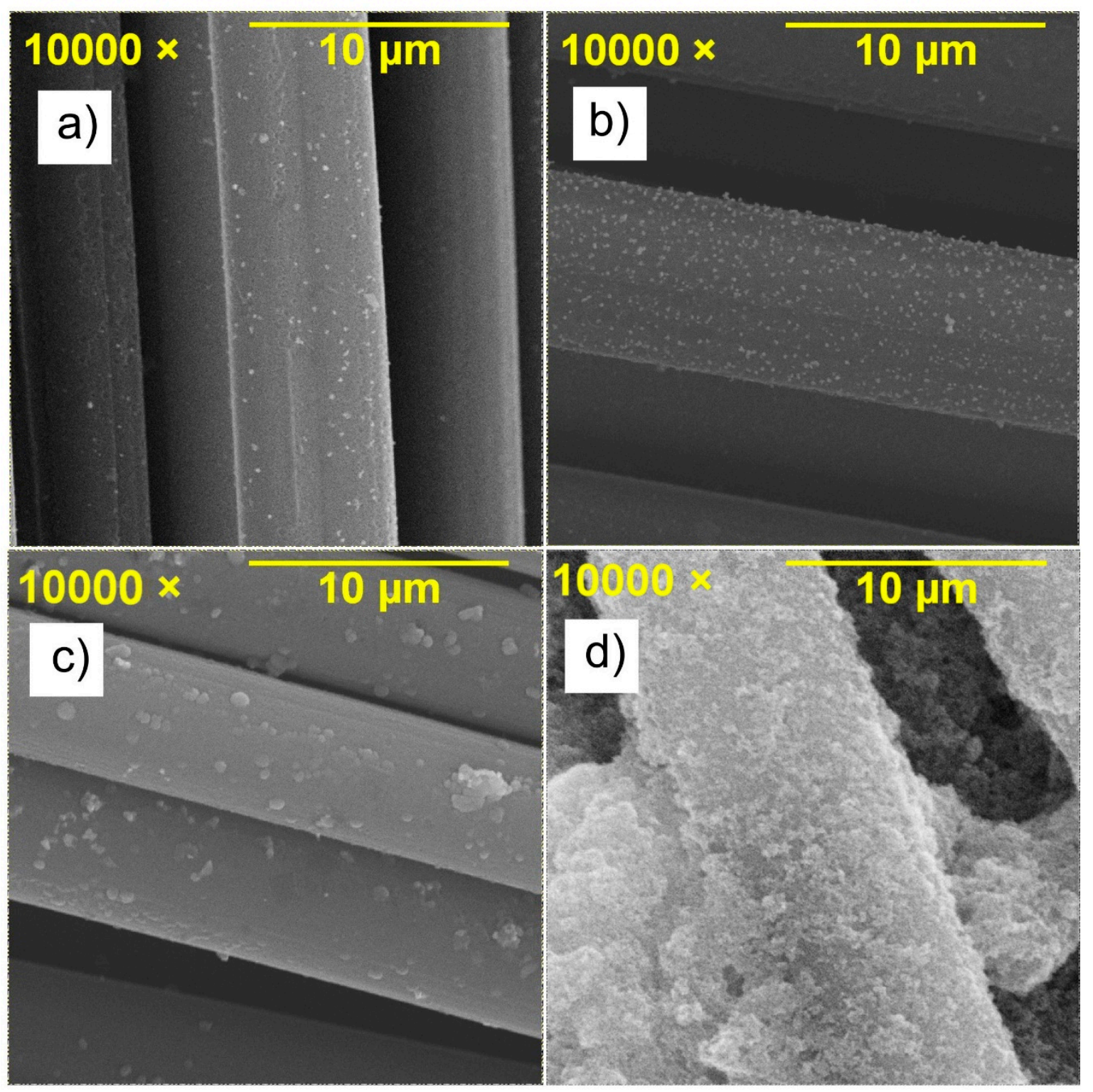

Figure 1. SEM images of the Busofit materials: (a) Basic; (b) with Ag spraying; (c) with Ti spraying; (d) with infiltrated dispersion of $\mathrm{TiO}_{2}$ nanoparticles. 
Figure $1 b, c$ show particles of deposited metal on the surface of the fiber. Figure $1 d$ shows a thick coating. These particles can form sorption centers for redox reactions and have an influence on the interaction between electrolytes and the Busofit surface.

Plots of the maximum power density versus temperature difference between the hot and cold sides of the cell for the CR2025 case and cells with a salt bridge are shown in Figure 2.
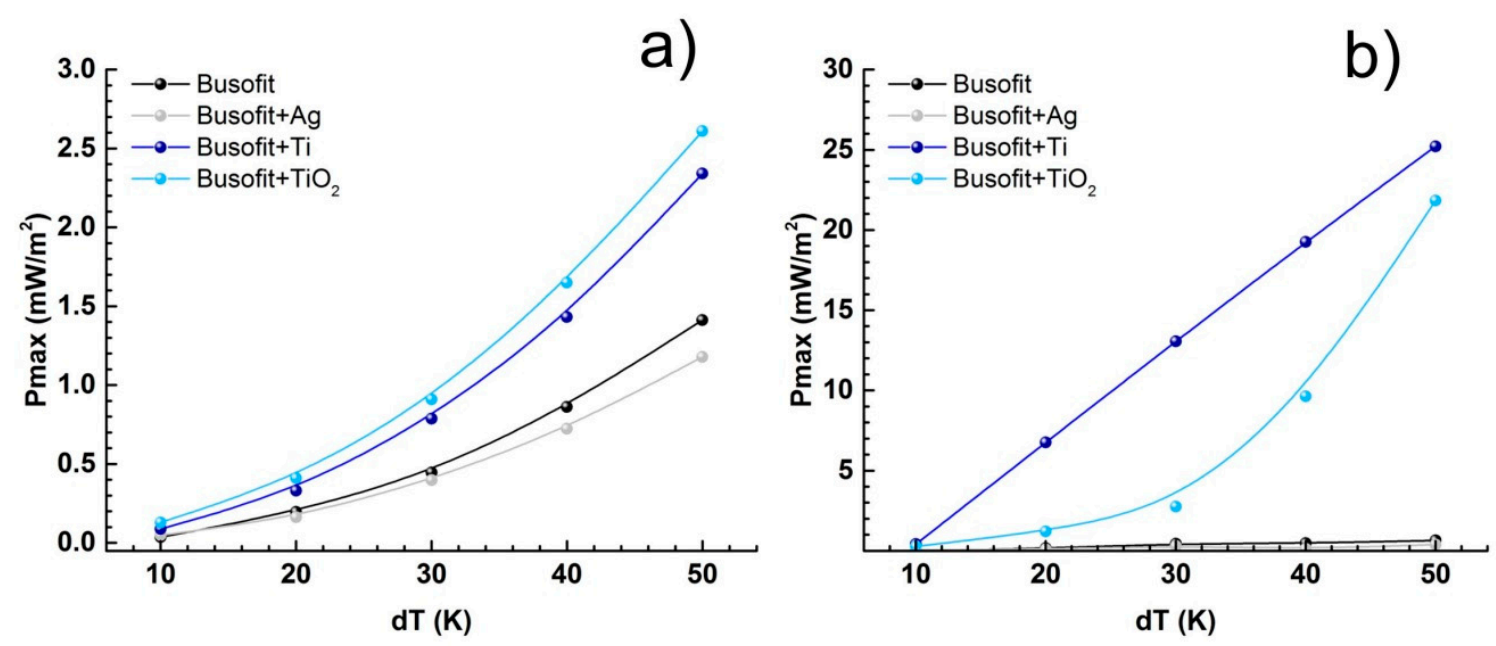

Figure 2. Plots of maximum power density versus temperature difference between the hot and cold sides of the cell (a) for cells with a salt bridge and (b) for cells in the coin cell CR2025.

The plots for the cells with a salt bridge (Figure 2a) show that the presence of the Ag modifier does not significantly affect the power of the system, while Ti deposition and $\mathrm{TiO}_{2}$ dispersion significantly increase the output power (by about two times). This effect may be attributed to the reduction in the interface resistance between the electrodes and the electrolyte because of the catalytic effect of $\mathrm{TiO}_{2}$ in the dispersion and the existence of oxide film on the surface of the deposited titanium.

In the coin cell case, the electrodes are very close to each other (about $1 \mathrm{~mm}$ apart); therefore, the actual temperature difference between the electrodes should be lower and the potential difference should also be lower. Nevertheless, the power output of the coin cell with the electrodes made of native Busofit and that of Busofit modified with Ag are close to the value obtained for the cells with a salt bridge. This can be explained by the lower resistance of the cell without a salt bridge and high exchange current values, due to the distance between the electrodes.

Based on the I-V plots of the thermal cells (Supplementary Materials Figures S1-S8), the values of the open circuit voltage and the short circuit current were compared (Table 1).

Table 1. The influence of surface modifier on the maximum values of $U_{\mathrm{OC}}$ and $\mathrm{I}_{\mathrm{SC}}$ for different types of thermo-electrochemical cells (TECs) (temperature difference between the hot and cold sides is $50 \mathrm{~K})$.

\begin{tabular}{ccccc}
\hline & \multicolumn{2}{c}{ Salt Bridge } & \multicolumn{2}{c}{ Coin Cell } \\
\cline { 2 - 5 } & $\mathbf{U}_{\mathbf{O C}}, \mathbf{~} \mathbf{~ V}$ & $\mathbf{I}_{\mathbf{S C}}, \mathbf{m k A}$ & $\mathbf{U}_{\mathbf{O C}}, \mathbf{m V}$ & $\mathbf{I}_{\mathbf{S C}}, \mathbf{m k A}$ \\
\hline Native Busofit & 71.5 & 12.0 & 11.1 & 51 \\
Busofit with $\mathrm{Ag}$ & 75.4 & 9.4 & 7.0 & 44 \\
Busofit with $\mathrm{Ti}$ & 75.4 & 17.7 & 11.3 & 1217 \\
Busofit with $\mathrm{TiO}_{2}$ & 72.6 & 21.5 & 19.0 & 682 \\
\hline
\end{tabular}

The values of the open circuit voltage of cells with a salt bridge for all Busofit types are relatively close (Table 1). In this case, the voltage of the open circuit can be used to calculate 
the actual temperature difference of the electrodes using the formula: $\Delta \mathrm{T}=\mathrm{U}_{\mathrm{oc}} / \mathrm{Se}$, where Se is $1.4 \mathrm{mV} / \mathrm{K}$.

For a coin cell, the real electrode temperature is significantly dependent on the thermal conductivity of the cell (electrodes, electrolyte, and current collector). Therefore, different Busofit modifications have different real temperature gradients, which are confirmed by $\mathrm{U}_{\mathrm{OC}}$. Hence, Busofit filled with $\mathrm{TiO}_{2}$ which has lower thermal conductivity than native carbon fiber and carbon fiber with metallic silver or titanium showed higher UOC $(19 \mathrm{mV})$ because of the higher real temperature gradient. The short circuit current is proportional to the equivalent serial resistance (ESR), which is obtained as the sum of the resistance of the electrolyte and the resistance to charge transfer through the electrode-electrolyte junction. It is obvious that the resistance of the electrolyte in coin cells is significantly lower than that of cells with a salt bridge, which is confirmed by the impedance data.

Nyquist plots (Figure 3a-e) have the shape of a semicircle and are equivalent to the parallel connection of capacitance and resistance [15]. In a TEC with an electrolyte containing Fe ions in the heterovalent state, the reaction proceeds on the surface of the hot electrode,

$$
\mathrm{Fe}(\mathrm{CN})_{6}{ }^{3-}-\mathrm{e}^{-} \rightarrow \mathrm{Fe}(\mathrm{CN})_{6}{ }^{4-}
$$

and on the surface of the cold electrode,

$$
\mathrm{Fe}(\mathrm{CN})_{6}{ }^{4-}+\mathrm{e}^{-} \rightarrow \mathrm{Fe}(\mathrm{CN})_{6}{ }^{3-}
$$

The impedance of a TEC consists of $\mathrm{R}_{\mathrm{s}}$ - electrolyte resistance; $\mathrm{CPE}_{\mathrm{dl}(\mathrm{c})}, \mathrm{CPE}_{\mathrm{dl}(\mathrm{h})}$ constant phase elements for cold and hot electrodes that correspond to capacities of an electrical double layer at the electrode-electrolyte interface. $R_{\mathrm{ct}(\mathrm{c})}$ and $\mathrm{R}_{\mathrm{ct}(\mathrm{h})}$ are the charge transfer resistance of cold electrode-electrolyte and hot electrode-electrolyte junctions, respectively.

The complete impedance circuit (Figure 3e) can be simplified provided that the resistances and capacitances of the electric double layer of the hot and cold electrodes differ by small values. During reactions (2) and (3), the potential of the electrodes for carbon materials changes. These changes are accompanied by the accumulation of an pseudo-intensity of redox reactions on the surface of the Busofit material:

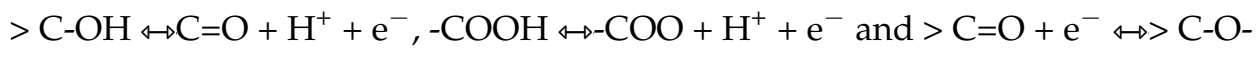

At a temperature gradient, the TEC gives off charge as a supercapacitor, i.e., the current is inversely proportional to the ESR, which is the sum of the resistance of the electrolyte and the resistance of the charge transfer of the electrodes. To confirm the hypothesis of limiting resistance and to calculate the thermoelectric conversion efficiency indicator, an analysis of the results by impedance spectroscopy was carried out using the method of determination parameters of the equivalent circuit. The equivalent scheme of the TEC and dependences of the cell's resistance are shown in Figure 3.

On basis of these results, the dependence of the resistance of the electrolyte on the temperature difference of the TEC was established.

The calculated values of the elements of the equivalent scheme (Figure 3a) are shown in Table 2. 

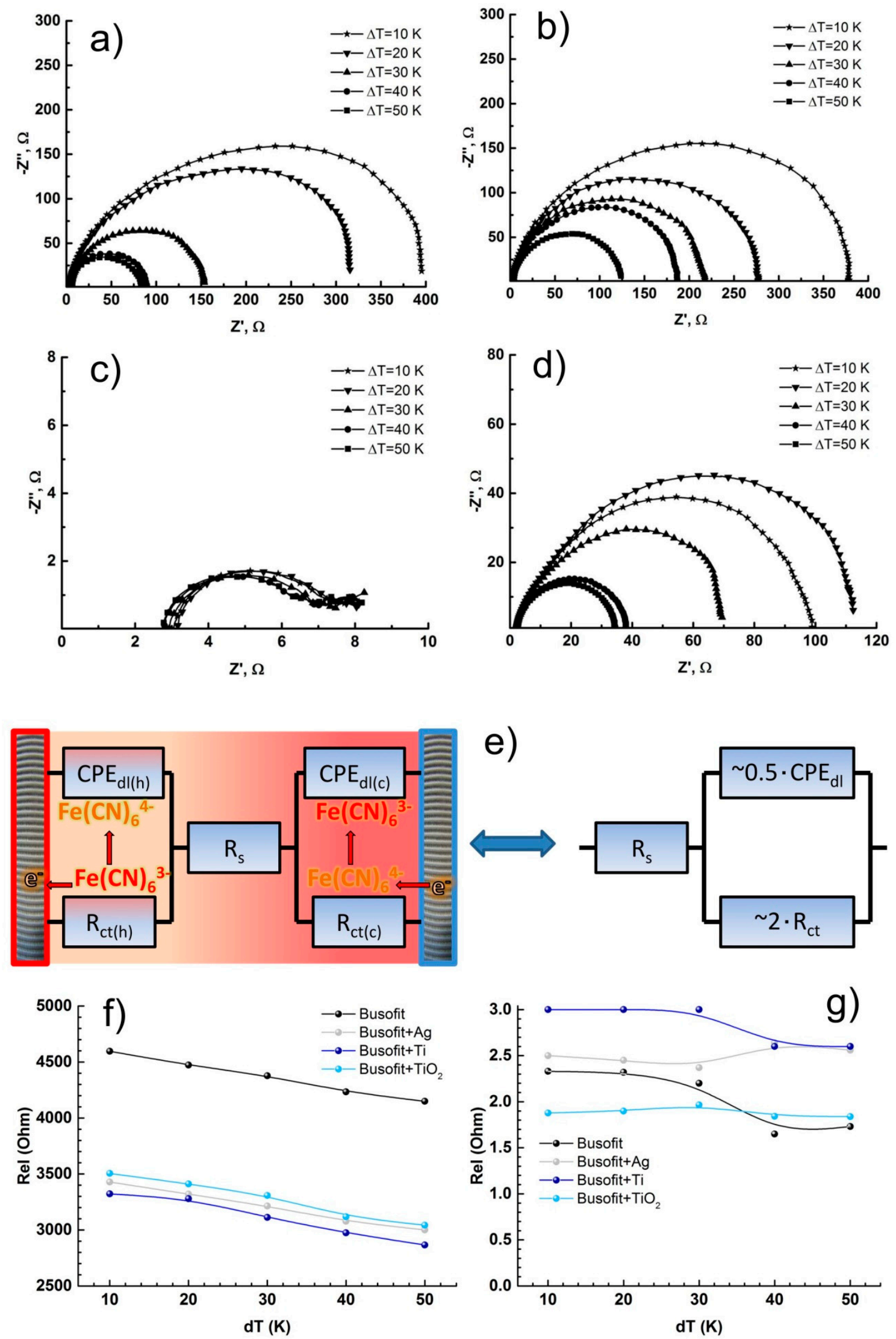

Figure 3. Nyquist plots for cells in a coin cell CR2025: (a) Busofit, (b) Busofit Ag, (c) Busofit Ti, and (d) Busofit TiO ${ }_{2}$; (e) equivalent scheme of a TEC (e); (f) the dependence of the resistance of the electrolyte on the temperature difference between the electrodes for cells with a salt bridge and (g) for cells in a coin cell CR2025. 
Table 2. Elements of the equivalent scheme of the studied TECs.

\begin{tabular}{cccc}
\hline Type of Electrodes & $\mathbf{R}_{\mathbf{s}}(\boldsymbol{\Omega})$ & $\mathbf{C}_{\mathbf{d l}}(\mathbf{m k F})$ & $\mathbf{R}_{\mathrm{ct}}(\boldsymbol{\Omega})$ \\
\hline Busofit (Salt bridge) & 4150 & 6 & 1100 \\
Busofit (Coin cell) & 2 & 3 & 80 \\
\hline Busofit+Ag (Salt bridge) & 3000 & 3 & 4090 \\
Busofit+Ag (Coin cell) & 2 & 3 & 120 \\
\hline Busofit+ $\mathrm{Ti}$ (Salt bridge) & 2870 & 3 & 201 \\
Busofit+Ti (Coin cell) & 2 & 10 & 5 \\
\hline Busofit+ $\mathrm{TiO}_{2}$ (Salt bridge) & 3040 & 6 & 30 \\
Busofit+ $\mathrm{TiO}_{2}$ (Coin cell) & 2 & 3 & 30 \\
\hline
\end{tabular}

From these results, it is seen that the resistance of the electrolyte in systems with a salt bridge is thrice that in a system in a coin cell CR2025. This explains the difference in the values of specific power and current density between systems with the same electrodes but assembled in different cell architectures.

A comparison of the resistance of the electrode process for various modifications of the Busofit fiber shows that the resistance of the fiber modified with titanium is 15 to 20 times lower than the resistance of the base fiber or the resistance of the one modified with silver. At the same time, fiber modified with titanium oxide powder has a higher resistance, but it is significantly lower than the starting material.

Such significant differences in resistance are reflected in parameters such as short circuit current and output power. On the one hand, the short circuit current at the maximum temperature gradient for cells in the coin cell was, on average, twice as high as that for cells with a salt bridge. In addition, the short circuit current of Busofit fibers modified with titanium ( $\mathrm{Ti}$ and $\mathrm{TiO}_{2}$ ) was about 20-30 times higher than that for base fibers or fibers with silver.

Busofit contact resistance consists of the internal resistance of individual fibers, the total resistance of each contact between the fibers, and the charge transfer resistance across the electrode-electrolyte junction. It is obvious that Busofit is in a compressed state, i.e., assembled in a coin-cell form, and has the lowest total resistance of all contacts between the fibers compared with the circuit with a bridge, as confirmed by the data in Table 1. In this case, the silver coating (sputtering) on the surface of Busofit increases the impedance of the contacts and is apparently associated with a rapid oxidation of silver and the formation of oxide with high resistance. In contrast, titanium sputtering significantly reduced contact resistance from 80 to $5 \Omega$ (Table 2).

On the other hand, the open circuit voltage (Supplementary Materials) for cells in the coin cell is lower, on average, by $5-15$ times compared to cells with a salt bridge, which is associated with a lower temperature gradient.

Thus, regardless of the type of cell architecture, modification of the electrode material with titanium or titanium oxide significantly improves the short circuit current of any type cell. This effect is associated with the catalytic effect of titanium oxide on the oxidation reaction occurring on the hot electrode.

To confirm the hypothesis of the catalytic effect of titanium dioxide on the surface of the electrode material Busofit, the resistance value of the electrode process $R_{c t}$ was analyzed. The dependence of the $R_{c t}$ value on temperature at the Arrhenius coordinates for the Busofit-based fiber systems with sputtered titanium, silver, and infiltrated titanium dioxide in the cells of the coin cell is shown in Figure 4a. The dependence of $R_{c t}$ on the absolute temperature at the Arrhenius coordinates is linear. The activation energy of the electrode process is depicted in the summary plot (Figure $4 \mathrm{~b}$ ). The activation energy of the electrode process in system with titanium dioxide is lower than in other systems. 

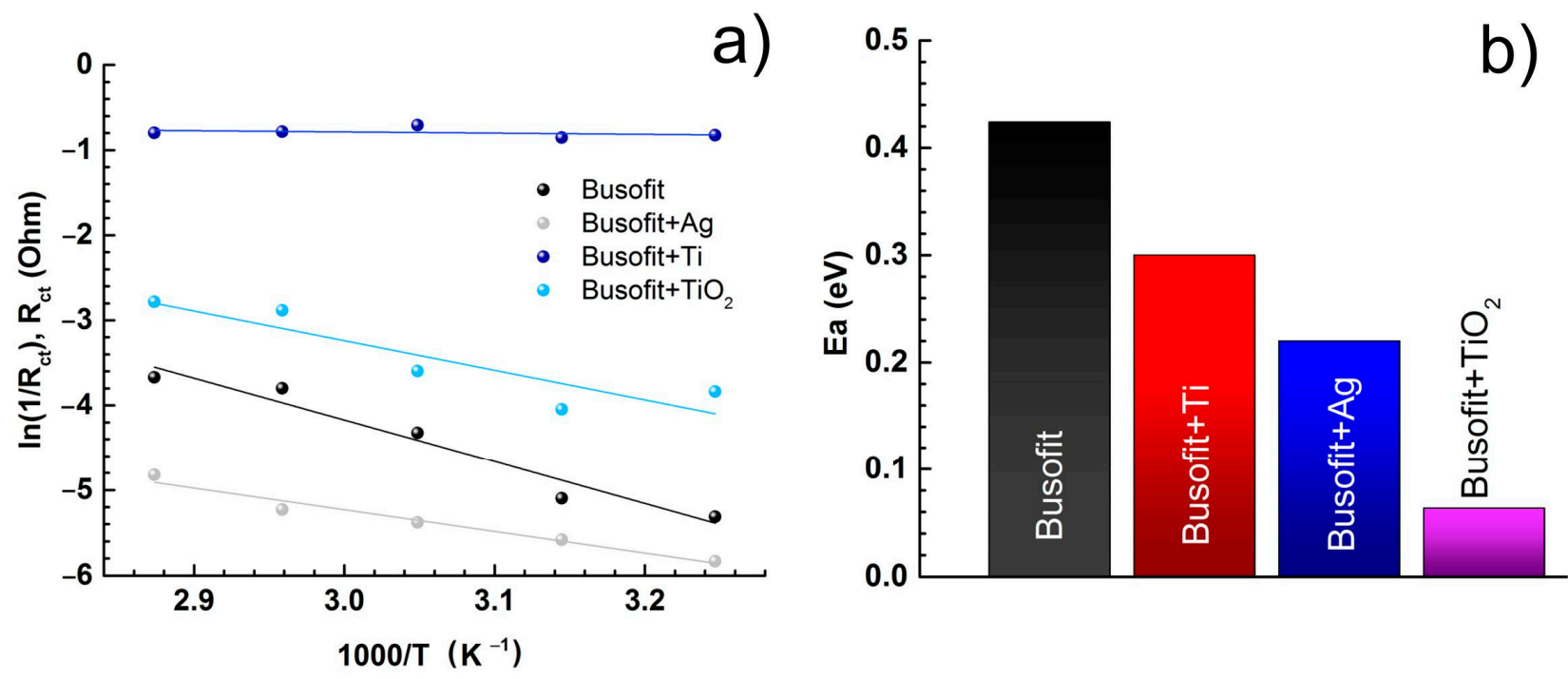

Figure 4. Dependence of the resistance of the electrode process on the temperature at the (a) Arrhenius coordinates, and (b) values of the activation energy of the electrode process in systems with the base fabric Busofit with Ag coating, Ti coating, and infiltrated $\mathrm{TiO}_{2}$.

The efficiency of thermoelectric conversion was calculated by the method in [26]. The dependence of the equivalent parameter $\mathrm{ZT}$ on the temperature gradient of the electrodes is shown in Figure 5. Thus, ZT varies with change in resistance.
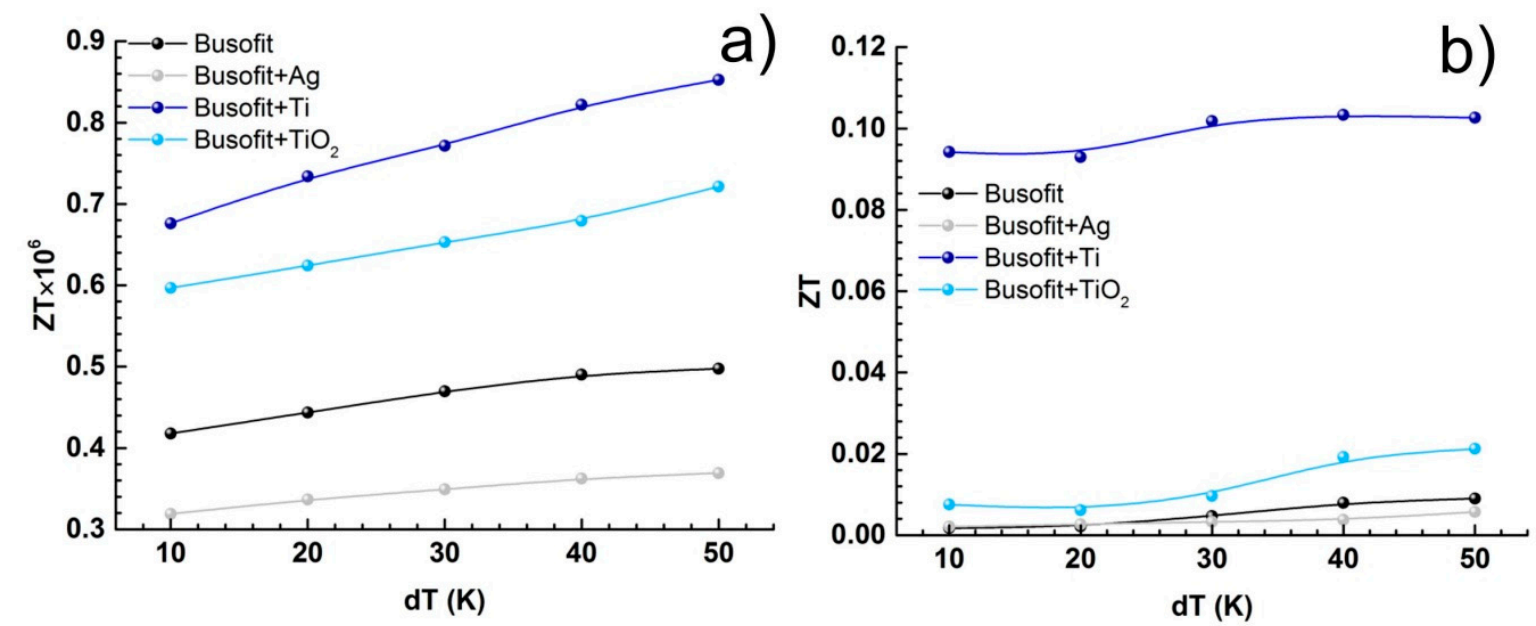

Figure 5. The dependence of the equivalent parameter ZT on the temperature gradient (a) for cells with a salt bridge and (b) for cells in a coin cell CR2025.

In [27], it was shown that the values of ZT typical for solid-state inorganic thermal generators are 1 or less at temperatures of about $800{ }^{\circ} \mathrm{C}$. For example, for $\mathrm{Bi}_{2} \mathrm{Ti}_{3}, \mathrm{ZT}$ is in the range of 0.5-0.8; for composites based on rare-earth metals, ZT is in the range of $0.75-1.2$, and for polymeric materials, ZT is in the range of $0.2-0.42$.

Based on the ZT values, the dependence of the thermoelectric conversion efficiency was established (Figure 6). Plots of the thermoelectric conversion efficiency reveal that the efficiency of the system with a salt bridge is very low. This can be explained by the high resistance of the electrolyte in the connecting tubes. The system with carbon fiber Busofit coated with titanium in a coin cell CR2025 showed the highest efficiency of $1.37 \%$, with a temperature difference of $50 \mathrm{~K}$. In a previously published review [4], values for typical thermo-electrochemical cells $<1 \%$ were shown and it was noted that an efficiency of $2-5 \%$ will be sufficient for applied use. 

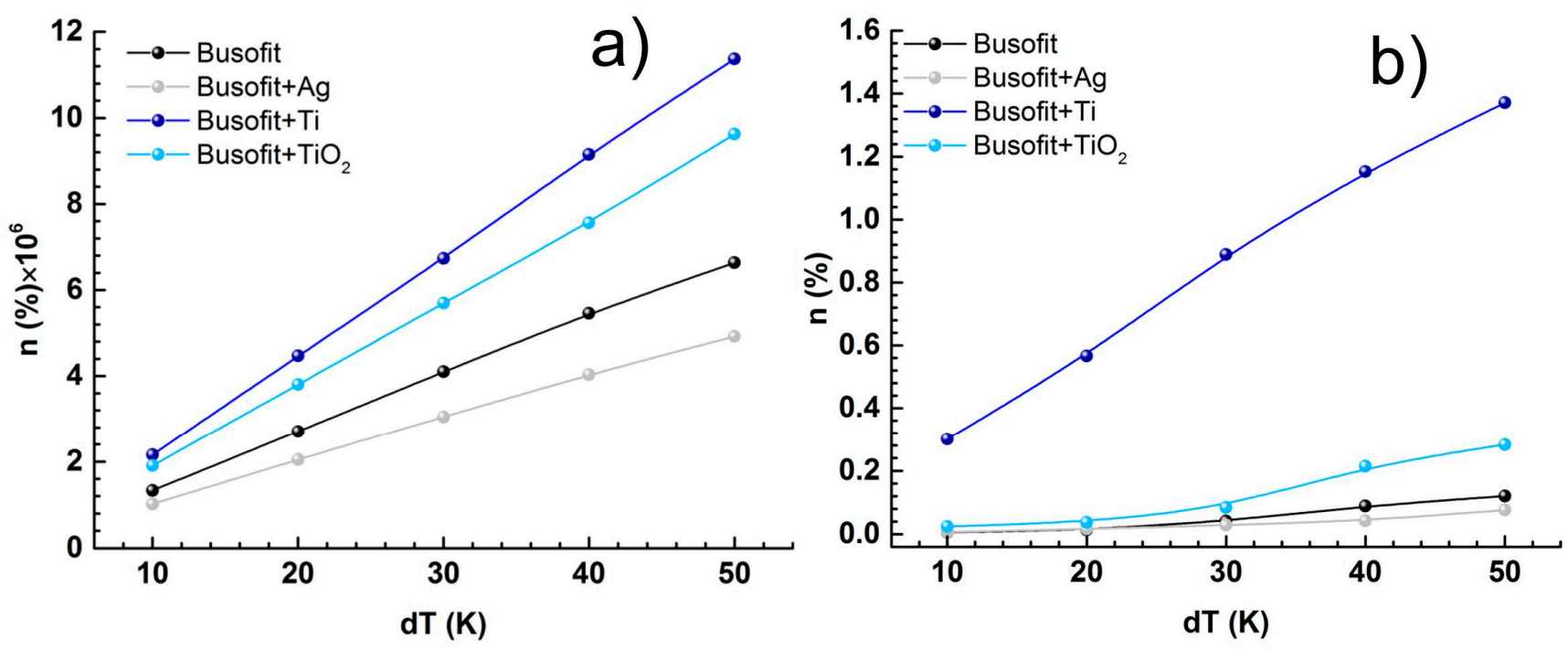

Figure 6. The dependence of the efficiency of the studied cells on the temperature gradient of the electrodes (a) for cells with a salt bridge and (b) for cells in a coin cell CR2025.

\section{Conclusions}

In this work, the properties of TECs with two different cell architectures (electrochemical cell with a salt bridge and a coin cell case) based on modified carbon fiber electrodes were studied. The electrochemical impedance of TECs for various temperature gradients was described by an equivalent scheme; the limiting impedance components for the salt bridge and coin cell systems were calculated and explained for three parameters $\left(R_{s}\right.$, $\mathrm{CPE}_{\mathrm{dl}(\mathrm{c})}, \mathrm{CPE}_{\mathrm{dl}(\mathrm{h})}, \mathrm{R}_{\mathrm{ct}(\mathrm{c})}$ and $\mathrm{R}_{\mathrm{ct}(\mathrm{h})}$, look at the Figure $\left.3 \mathrm{e}\right)$.

Close distance between electrodes causes low values of electrolyte resistance in the coin cell. At the same time, different thermal conductivity of carbon fibers modified with $\mathrm{Ag}, \mathrm{Ti}$, and $\mathrm{TiO}_{2}$ causes different real temperature in the electrode-electrode contact zone and significantly decreases the real temperature gradient. This effect is well observed from the dependence of the open circuit voltage $\left(\mathrm{U}_{\mathrm{OC}}\right)$ on the type of modifier in the cell in Table 1, which is significantly higher for cells with a salt bridge. Nevertheless, high resistance of the salt bridge decreases the exchange current, and for this reason, the relative efficiency and power output of the coin cell system exceed the values of these parameters in cells with a salt bridge.

The catalytic effect of the Busofit modifiers was confirmed by a change in the activation energies, which were calculated from the Arrhenius dependences for contact resistance. The $\mathrm{TiO}_{2}$ Busofit modifier, with an activation energy about $0.3 \mathrm{eV}$, has the greatest catalytic effect. The highest value of specific electric power of the cells was achieved in the coin cell system with Busofit+Ti electrodes. This can be caused by the combination of the high conductivity of titanium metal, which reduces the resistance between individual carbon filaments, and the catalytic effect of a thin oxide film on its surface. The values of the specific power and the efficiency were $25.2 \mathrm{~mW} / \mathrm{m}^{2}$ and $1.37 \%$, respectively.

Supplementary Materials: The following are available online at https:/ /www.mdpi.com/2071-105 0/13/3/1377/s1, Figure S1: (a) I-V curves and (b) P-V curves for cells with salt bridge and basic.fiber Busofit respectively; Figure S2: (a) I-V curves and (b) P-V curves for cells with salt bridge and Ag-sputtering Busofit respectively; Figure S3: (a) I-V curves and (b) P-V curves for cells with salt bridge and Ti-sputtering Busofit re-spectively; Figure S4: (a) I-V curves and (b) P-V curves for cells with salt bridge and Busofit with infiltrated dispersion of $\mathrm{TiO}_{2}$ nanoparticles respectively; Figure S5: (a) I-V curves and (b) P-V curves for cells in CR 2025 case and basic fiber Busofit re-spectively; Figure S6: (a) I-V curves and (b) P-V curves for cells in CR 2025 case and Ag-sputtering Busofit respectively; Figure S7: (a) I-V curves and (b) P-V curves for cells in CR 2025 case and Ti-sputtering 
Busofit re-spectively; Figure S8: (a) I-V curves and (b) P-V curves for cells in CR 2025 case and Busofit with infiltrated dispersion of $\mathrm{TiO}_{2}$ nanoparticles respectively.

Author Contributions: Conceptualization and methodology, N.G.; validation, N.K. (Natalya Kovyneva) and M.V.; formal analysis, E.B.; software and visualization, D.A.; investigation, O.G.; resources, A.G.; data curation and writing-original draft preparation, N.K. (Nikolay Kiselev); writing-review and editing, P.O.; supervision and project administration, I.B. All authors have read and agreed to the published version of the manuscript.

Funding: This research received no external funding.

Institutional Review Board Statement: Not applicable.

Informed Consent Statement: Not applicable.

Data Availability Statement: Not applicable.

Conflicts of Interest: The authors declare no conflict of interest.

\section{References}

1. Im, H.; Kim, T.; Song, H.; Choi, J.; Park, J.S.; Ovalle-Robles, R.; Yang, H.D.; Kihm, K.D.; Baughman, R.H.; Lee, H.H.; et al. Highefficiency electrochemical thermal energy harvester using carbon nanotube aerogel sheet electrodes. Nat. Commun. 2016, 7, 10600. [CrossRef]

2. Dupont, M.F.; Macfarlane, D.R.; Pringle, J. Thermo-electrochemical cells for waste heat harvesting-Progress and perspectives. Chem. Commun. 2017, 53, 6288-6302. [CrossRef]

3. Burmistrov, I.; Kovyneva, N.; Gorshkov, N.; Gorokhovsky, A.; Durakov, A.; Artyukhov, D.; Kiselev, N. Development of new electrode materials for thermo-electrochemical cells for waste heat harvesting. Renew. Energy Focus 2019, 29, 42-48. [CrossRef]

4. Zhang, L.; Kim, T.; Li, N.; Kang, T.J.; Chen, J.; Pringle, J.M.; Zhang, M.; Kazim, A.H.; Fang, S.; Haines, C.; et al. High Power Density Electrochemical Thermocells for Inexpensively Harvesting Low-Grade Thermal Energy. Adv. Mater. 2017, $29,1605652$. [CrossRef]

5. Romano, M.S.; Li, N.; Antiohos, D.; Razal, J.M.; Nattestad, A.; Beirne, S.; Fang, S.; Chen, Y.; Jalili, R.; Wallace, G.G.; et al. Carbon Nanotube-Reduced Graphene Oxide Composites for Thermal Energy Harvesting Applications. Adv. Mater. 2013, 25, 6602-6606. [CrossRef]

6. Zhou, Y.; Qian, W.; Huang, W.; Liu, B.; Lin, H.; Dong, C. Carbon Nanotube-Graphene Hybrid Electrodes with Enhanced Thermo-Electrochemical Cell Properties. Nanomaterials 2019, 9, 1450. [CrossRef]

7. Hirai, T.; Shindo, K.; Ogata, T. Charge and Discharge Characteristics of Thermochargeable Galvanic Cells with an $\left[\mathrm{Fe}(\mathrm{CN})_{6}\right]^{4-} /\left[\mathrm{Fe}(\mathrm{CN})_{6}\right]^{3-}$ Redox Couple. J. Electrochem. Soc. 1996, 143, 1305. [CrossRef]

8. Artyukhov, D.; Kiselev, N.; Gorshkov, N.; Burmistrov, I. Research of the influence of electrolyte concentration on ther-moelectrochemical cells efficiency. Proc. Environ. Sci. Eng. Manag. 2019, 6, 319-327.

9. Duan, J.; Feng, G.; Yu, B.; Li, J.; Chen, M.; Yang, P.; Feng, J.; Liu, K.; Zhou, J. Aqueous thermogalvanic cells with a high Seebeck coefficient for low-grade heat harvest. Nat. Commun. 2018, 9, 1-8. [CrossRef]

10. Hu, R.; Cola, B.A.; Haram, N.; Barisci, J.N.; Lee, S.; Stoughton, S.; Wallace, G.; Too, C.; Thomas, M.; Gestos, A.; et al. Harvesting Waste Thermal Energy Using a Carbon-Nanotube-Based Thermo-Electrochemical Cell. Nano Lett. 2010, 10, 838-846. [CrossRef]

11. Salazar, P.F.; Kumar, S.; Cola, B.A. Design and optimization of thermo-electrochemical cells. J. Appl. Electrochem. 2013, 44, 325-336. [CrossRef]

12. Wu, J.; Black, J.J.; Aldous, L. Thermoelectrochemistry using conventional and novel gelled electrolytes in heat-to-current thermocells. Electrochim. Acta 2017, 225, 482-492. [CrossRef]

13. Zhou, H.; Liu, P. High Seebeck Coefficient Electrochemical Thermocells for Efficient Waste Heat Recovery. ACS Appl. Energy Mater. 2018, 1, 1424-1428. [CrossRef]

14. Burmistrov, I.; Gorshkov, N.; Kovyneva, N.; Kolesnikov, E.; Khaidarov, B.; Karunakaran, G.; Cho, E.-B.; Kiselev, N.; Artyukhov, D.; Kuznetsov, D.; et al. High seebeck coefficient thermo-electrochemical cell using nickel hollow microspheres electrodes. Renew. Energy 2020, 157, 1-8. [CrossRef]

15. Burmistrov, I.; Gorshkov, N.; Kiselev, N.; Artyukhov, D.; Kolesnikov, E.; Khaidarov, B.; Yudni, A.; Karunakaran, G.; Cho, E.-B.; Kuznetsov, D.; et al. Data on the current-voltage dependents of nickel hollow microspheres based thermo-electrochemical in alkaline electrolyte. Data Brief 2020, 31, 105770. [CrossRef]

16. Gunawan, A.; Lin, C.-H.; Buttry, D.A.; Mujica, V.; Taylor, R.A.; Prasher, R.; Phelan, P. Liquid Thermoelectrics: Review of Recent and Limited New Data of Thermogalvanic Cell Experiments. Nanoscale Microscale Thermophys. Eng. 2013, 17, 304-323. [CrossRef]

17. Benji, M.; Alam, K. Carbon nanotubes and nanofibers in composite materials. Sampe J. 2002, 38, 59-70.

18. Miyasaka, K.; Watanabe, K.; Jojima, E.; Aida, H.; Sumita, M.; Ishikawa, K. Electrical conductivity of carbon-polymer composites as a function of carbon content. J. Mater. Sci. 1982, 17, 1610-1616. [CrossRef]

19. Feng, L.; Xie, N.; Zhong, J. Carbon Nanofibers and Their Composites: A Review of Synthesizing, Properties and Applications. Materials 2014, 7, 3919-3945. [CrossRef] 
20. Salazar, P.F.; Kumar, S.; Cola, B.A. Nitrogen- and Boron-Doped Carbon Nanotube Electrodes in a Thermo-Electrochemical Cell. J. Electrochem. Soc. 2012, 159, B483-B488. [CrossRef]

21. Alzahrani, H.A.; Buckingham, M.A.; Marken, F.; Aldous, L. Success and failure in the incorporation of gold nanoparticles inside ferri/ferrocyanide thermogalvanic cells. Electrochem. Commun. 2019, 102, 41-45. [CrossRef]

22. Gong, K.; Du, F.; Xia, Z.; Durstock, M.; Dai, L. Nitrogen-Doped Carbon Nanotube Arrays with High Electrocatalytic Activity for Oxygen Reduction. Science 2009, 323, 760-764. [CrossRef]

23. Bae, K.M.; Yang, H.D.; Tufa, L.T.; Kang, T.J. Thermobattery based on CNT coated carbon textile and thermoelectric electrolyte. Int. J. Precis. Eng. Manuf. 2015, 16, 1245-1250. [CrossRef]

24. Im, H.; Moon, H.G.; Lee, J.S.; Chung, I.Y.; Kang, T.J.; Kim, Y.H. Flexible thermocells for utilization of body heat. Nano Res. 2014, 7, 443-452. [CrossRef]

25. Macdonald, J.R.; Barsoukov, E. Impedance Spectroscopy: Theory; Experiment; and Applications, 2nd ed.; Wiley \& Sons: New York, NY, USA, 2005; pp. 289-305.

26. Goldsmid, H.J. Introduction to Thermoelectricity; Springer: Berlin, Germany, 2010; p. 46.

27. Champier, D. Thermoelectric generators: A review of applications. Energy Convers. Manag. 2017, 140, 167-181. [CrossRef] 